\title{
TRADUCTORAS GALLEGAS DEL SIGLO XX: REESCRIBIENDO LA HISTORIA DE LA TRADUCCIÓN DESDE EL GÉNERO Y LA NACIÓN
}

\author{
Olga Castro \\ University of Exeter
}

\section{Resumen}

Dentro de los incipientes Estudios Gallegos, la crítica feminista ha mostrado un empeño en desvelar el papel de las mujeres en el desarrollo del polisistema cultural. Sin embargo, en la historiografía feminista gallega la faceta traductora de muchas mujeres todavía no resulta suficientemente reconocida. Es por ello que, en el marco del creciente interés por desvelar el papel que han desempeñado en la historia de la humanidad tanto la traducción como las mujeres, este artículo busca explorar la historia de la traducción del siglo XX en el contexto gallego para poner de relieve la contribución de las traductoras, con un doble propósito: por un lado, ofrecer unas pinceladas sobre algunas traductoras como Mercedes Vázquez Fernández Pimentel, Mari Luz Morales, Teruca Bouza Vila, María Barbeito, Amparo Alvajar, Xohana Torres o Teresa Barro, abriendo nuevos campos de investigación que en el futuro puedan ser examinados en mayor profundidad. Por el otro, analizar las relaciones de poder presentes en su labor traductora a la luz del discurso del género y de la nación.

\begin{abstract}
"Galician Women Translators in the $20^{\text {th }}$ Century: Rewriting the History of Translation from a Gender and National Approach"

Within the newly established field of Galician Studies, Feminist Theory has played a major role in revealing how women have contributed to the development of the Galician cultural polysystem. However, it is my contention that the translative facet of many women translators has not yet received enough critical attention. Therefore, within the framework of a growing interest in the roles played both by women and by translation in the development of societies, this article seeks to explore the history of translation in the context of Galicia, with a view to underscoring the contributions of
\end{abstract}


women translators throughout the $20^{\text {th }}$ century. The aim of the article is twofold: firstly, to offer an overview of translators such as Mercedes Vázquez Fernández Pimentel, Mari Luz Morales, Teruca Bouza Vila, María Barbeito, Amparo Alvajar, Xohana Torres, and Teresa Barro, in order to open up new areas for research so that subsequent studies can further examine their contributions in more depth. Secondly, it seeks to analyse the power relations which inform the activity of translation both from a gender and national approach.

Palabras clave: Traductoras gallegas. Historiografía feminista de la traducción, género y nación. Traducción gallega del siglo XX.

Keywords: Galician women translators. Feminist historiography of translation, gender and nation. Galician translation in the 20th century. 
Hace ya casi una década, el experto en historia de la traducción Jean Delisle (2002a: 8) advertía un creciente interés por reconstruir la genealogía feminista de la práctica y teoría de la traducción encaminado, sobre todo, a la recuperación de figuras relevantes de traductoras y teóricas. En efecto, diferentes congresos sobre el papel de las traductoras en la historia celebrados en los últimos años atestiguan este interés, a los que han seguido numerosos artículos de investigación publicados en revistas académicas e incluso monografías como Portraits de traductrices, editada por el mismo Delisle (2002b) y ganadora del premio otorgado por la Canadian Association of Translation Studies al mejor libro del año. Es en este contexto en el que se ubica mi propuesta de reescribir la historia de la traducción en Galicia desde el discurso del género y de la nación, con el propósito de poner en valor el papel de las traductoras gallegas del siglo XX.

Recuperar el papel de las traductoras en volúmenes específicos sobre el tema permite corregir las exclusiones y otorgar visibilidad a estas figuras traductoras, como una forma de contrarrestar que los recopilatorios generalistas realizados hasta el momento se hayan centrado en exclusiva en la genealogía masculina. Ahora bien, restringirse a esta metodología compensatoria podría contribuir a afianzar todavía más la idea de que el universo mujeril es un gueto particular, como podría igualmente acabar sirviendo de excusa para que las llamadas investigaciones u obras generalistas continúen eludiendo su responsabilidad de género. Por esta razón, al lado de monografías y estudios específicos todavía muy necesarios (como el presente volumen), a mi juicio debe asimismo propiciarse que las traductoras y teóricas de la traducción figuren en recopilaciones generales, mencionadas en el período histórico o en la temática de contribución que les corresponde, como también en artículos que formen parte de publicaciones generalistas no dedicadas específicamente a mujeres.

\section{El doble compromiso de la historiografía feminista de la traducción}

El pasado otorga legitimidad. Es por ello que aquellos trabajos enmarcados en lo que podría denominarse como historiografía feminista de la traducción 
contribuyen al enriquecimiento y revalorización tanto de la traductología como de los estudios feministas, participando así de un doble compromiso. En el primer sentido, y dada la juventud de la disciplina traductológica como cuerpo autónomo e independiente, recuperar el pasado de la traducción contribuye a satisfacer "le besoin de légitimation d'une discipline nouvelle" (Lambert 1993: 22). Es más, dado el carácter de mediación intercultural inherente a todo acto de traducción, estos estudios contribuyen también a reivindicar para la traducción una posición más central en la historia de las sociedades y de la cultura que aquélla a la que está habitual y actualmente relegada (Lefevere 1992: xiv), participando así del argumento de que, como sostiene Henri van Hoof en Histoire de la traduction en Occident, "étudier l'histoire de la traduction, en effet, équivaut en quelque sorte à reprendre l'historie du monde, l'historie des civilisations, mais par le biais de la traduction" (van Hoof 1991: 7). Quizás aquí resida la razón de que el ritmo de publicaciones de carácter histórico sobre la traducción y traductología se haya acelerado considerablemente en el último cuarto de siglo (Lafarga 2005: 1133).

Respecto al segundo sentido, estos trabajos se enmarcan dentro de las contribuciones feministas a la historiografía, que desde los años 70 vienen cuestionando el supuesto carácter objetivo y neutro de la Historia y demostrando cómo las mujeres quedan tradicionalmente excluidas del relato oficial de la humanidad, construido en base a unas categorías conceptuales definidas por un marcado carácter androcéntrico (Lerner 1979). Frente a esta constatación, la historiografía feminista se marca como objetivo, en palabras de Joan Kelly-Gadol, "to restore women to history and to restore our history to women" (1976: 809), esto es, promover una reconstrucción de la memoria colectiva más inclusiva en la que se dé cuenta también de las contribuciones de las mujeres en sus propias voces y desde sus múltiples posiciones. Con este fin se han elaborado trabajos no sólo para difundir las aportaciones de mujeres singulares cuyos logros permanecían en la sombra (recuperando espacios de libertad de las mujeres en la esfera pública y creando unos precedentes para generaciones futuras), sino también para reconstruir el papel de las mujeres como grupo social en la historia y revalorizar la importancia de actividades consideradas típicamente femeninas.

La tradicional consideración de la traducción como una actividad femenina y reproductiva, en contraposición a la actividad de la escritura considerada productiva/creativa $y$, por tanto, masculina, favoreció que muchas mujeres que habían sido excluidas del mundo literario como autoras encontrasen en la traducción una válvula de escape. Y así, "c'est en grande partie la traduction qui leur a fourni ce moyen d'action et d'affirmation, les femmes n'étant 
alors autorisées à pénétrer dans le monde des idées que comme traductrices" (Delisle 2002a: 9-10). Es más, en algunos casos la traducción se convirtió en una de las pocas posibilidades para las mujeres de ejercer un empleo remunerado, hasta el punto de que "in some cases [it] was even the basis for financial independence from male relatives and as such a form of emancipation of the typical female role in the $18^{\text {th }}$ century society" (Wolf 2005: 17).

Ahora bien, aún siendo cierto que la traducción actuó como instrumento liberador al permitir el acceso de estas mujeres al mundo literario como traductoras, por el otro lado funcionó también como mecanismo opresor, condenándolas al margen del discurso. He aquí el código metonímico de doble inferioridad entre mujeres y traducción identificado por Lori Chamberlain (1988: 465), según el cual la diferencia de valor entre el original y la traducción puede explicarse tradicionalmente en paralelo a la diferencia de valor entre lo masculino y lo femenino. En consonancia con estos hallazgos, una constante en los estudios ha sido desvelar y poner en valor la contribución secular de las traductoras a la disciplina y a la práctica de la traducción, contribuyendo de este modo a reelaborar la genealogía de la traducción desde un punto de vista feminista.

No obstante, resulta llamativo y ciertamente cuestionable el rechazo explícito al calificativo 'feminista' en algunas de estas obras. Por ejemplo, en la ya mencionada Portraits de traductrices, el editor presenta la obra afirmando que

[1]e présent recueil n'est pas pour autant un ouvrage féministe. Il ne cherche pas à stigmatiser des injustices historiques dont les traductrices auraient été victimes et ne se veut pas non plus une dénonciation de leur marginalisation. It s'agit plutôt de mini-biographies de femmes qui ont consacré leur vie ou une partie de leur vie à la traduction et qui méritaient d'être mieux connues. (Delisle 2002a: 8-9)

A este respecto cabe apuntar que la revisión de la historia desvelando el papel de las mujeres es en sí mismo un objetivo feminista, independientemente de que las propias protagonistas mostrasen (o no) una consciencia de género sobre su situación. La contradicción en la que incurre este autor resulta todavía mayor cuando más adelante sostiene, a propósito de las traductoras estudiadas en el volumen, que

toutes ont, avec détermination, démontré aux hommes par leurs travaux intellectuels qu'être femme n'est pas un défaut. Refusant d'accepter les limites que la societé leur assignait, elles ont cherché, à leur manière, à briser le consensus des idées reçues à leur égard, à modifier, comme l'écrit Annie Brisset, "le regard des hommes sur les compétences intellectuelles des femmes". (Delisle 2002a: 9) 


\section{La historia de las mujeres en la traducción: principales líneas de la investigación}

El principal eje alrededor del que gravitan estos trabajos es el papel de las traductoras en el desarrollo de las sociedades en tanto que mediadoras interculturales, con capacidad para introducir nuevos debates sociales mediante la selección de determinadas obras para traducir. Así, muchas traductoras influyeron en el desarrollo de los movimientos sociales, culturales e intelectuales, contribuyendo al progreso científico, a la propagación de las religiones, a la exportación de los valores culturales y literarios, a la consolidación de las identidades nacionales o a la difusión del conocimiento, también del feminista. A este respecto, además de las traductoras estudiadas en el ya aludido volumen Portraits de traductrices editado por Delisle (2002b), en el territorio peninsular contamos con ejemplos de traductoras que contribuyeron de forma directa a difundir nuevas visiones sobre las relaciones de género mediante la traducción de obras feministas, como el caso de la gallega Emilia Pardo Bazán, que a finales del siglo XIX se convirtió en la traductora a castellano peninsular de la obra The Subjection of Women, hito del feminismo sufragista británico a cargo del filósofo John Stuart Mill. Una concepción similar de la traducción como mecanismo de subversión social feminista se encuentra en Carmen De Burgos, que a principios del siglo XX tradujo a castellano la obra La debilidad mental fisiológica de las mujeres, de su coetáneo y reconocido neurólogo austríaco Julius Moenius. La intención de De Burgos no era, obviamente, contribuir a la difusión de las ideas misóginas del original, sino utilizar la traducción como instrumento para rebatirlas. Así, su traducción incorpora un prólogo y numerosos pies de página con notas de la traductora en los que refuta el texto, como también lo hace en la serie de ensayos de su propia autoría que anexa a la traducción para dejar constancia de las condiciones de las mujeres de su época (cf. Sánchez 2010). Una tendencia semejante puede observarse respecto del feminismo literario, aspecto éste que ya ha sido ampliamente estudiado en artículos panorámicos sobre el caso catalán (cf. Godayol 2006 y 2007). También el ámbito catalán fue el primero de la península en contar con la obra de referencia de Simone de Beauvoir Le deuxième sexe (1949), gracias a la traducción en 1968 de Hermínia Grau y Carme Vilaginés. (A este respecto, cabe destacar que la obra sólo se tradujo a castellano peninsular en 1998, a cargo de Alicia Martorell, y a gallego en 2008, traducida por Marga Rodríguez Marcuño, cf. Castro Vázquez 2009).

Además de esto, un segundo eje en torno al que giran estos estudios es el papel de algunas de estas traductoras en tanto que teóricas de la traducción, por haber contribuido al discurso teórico de la disciplina con sus 
consideraciones en prefacios, dedicatorias, notas a pie de página o correspondencias personales donde reflexionaban sobre el acto de traducir, sobre los dilemas que les provocaba la traducción y sobre las limitaciones que condicionaban su práctica. De la importancia de recuperar estos metatextos ya ha dejado constancia (y ejemplo) Douglas Robinson, aduciendo que sería un primer paso para dejar de creer "that translation theory first begins to be written by women in the 1970s [...] and that wild, woolly, deviant translation theories are a (late-)twentieth-century phenomenon" (Robinson 1995: 172).

En tercer lugar, la vida de estas mujeres en la esfera pública que era la traducción con frecuencia estaba unida a la discriminación, pues aunque la traducción en algunos momentos históricos se considerase una actividad reproductiva (femenina), existían expectativas sociales patriarcales sobre qué tipo de obras las mujeres podían traducir y sobre cómo debían traducirlas, como existían también expectativas que censuraban que las traductoras asumiesen un yo autorial (masculino) desde el que reflexionar sobre su labor. Justamente por transgredir las expectativas sociales, y conscientes de que sus traducciones de autores de renombre serían infravaloradas y desprestigiadas, muchas traductoras adoptaron diferentes mecanismos para esconder su condición de mujer. ${ }^{1}$ Algunas lo hicieron recurriendo al anonimato, como Albertine Necker de Saussure en el siglo XVIII en sus traducciones de von Schlegel (Delisle 2002c: 117), o como Jane Wilde en el siglo XIX respecto a sus traducciones de Dumas, Meinhold o Lamartine (Cronin 2002: 266). Otras ocultaron su labor mediadora bajo un pseudónimo masculino, como Therese Huber, quien a finales del siglo XVIII y comienzos del XIX firmó sus traducciones con el nombre de su marido, siendo plenamente consciente de las expectativas sexistas sobre el trabajo de las mujeres. En este sentido, Huber denunció las prácticas de explotación laboral de las traductoras, que además de no ser pagadas directamente, sólo podían dedicarse a traducir tras finalizar con sus "deberes" domésticos (Wolf 2005: 20). Junto a ella, muchas otras traductoras eran conscientes de estas limitaciones de género, y por ello su propia labor traductora supuso, con frecuencia, todo un acto de subversión (feminista).

\section{El contexto gallego: particularidades del siglo XX}

Examinar en concreto el papel de las traductoras gallegas en el siglo pasado exige, previamente, abordar el contexto sociopolítico y las particularidades

1. Paradójicamente, en otros casos la traducción se convirtió para algunas escritoras en una manera de encubrir su autoría, que de esta forma firmaban sus originales como traducciones (cf. Kord 1994: 12). 
de un sistema cultural (literario, lingüístico) en el que se enmarca el ejercicio de su labor traductora. Dentro de lo que puede denominarse perspectiva occidental sobre la historia de la traducción, las aportaciones realizadas desde el sistema gallego están marcadas por su situación periférica, en tanto que colectivo minoritario y minorizado ubicado en los países del primer mundo. De hecho, esta consciencia de periferia está muy presente en la propuesta inaugural de Xoán González-Millán (1995) de articular, desde un plano teórico y para el caso gallego, una teoría de la traducción para sistemas marginales en la que la cuestión de la alteridad se erige como elemento diferenciador. Con una clara influencia de las teorías poscoloniales sobre la traducción y de la teoría de los Polisistemas, este autor sostiene que al percibir el acto de traducción como una lectura cultural de un texto empleando lenguas con estatutos culturales diferentes,

ábreselle a porta a unha teoría da tradución baseada na experiencia social da desigualdade $[\ldots]$ que valore a influencia do poder sobre os diferentes procesos de comunicación, superando os paradigmas que estabelecen un diálogo cultural en termos de harmoniosa igualdade. (González-Millán 1995: 65-66)

Con todo, esta consciencia de alteridad ya existía de forma latente en el plano práctico de la traducción en Galicia. Como evidencia el estudio panorámico de Camiño Noia (1995: 13), desde los inicios de la publicación en gallego en la Edad Media la traducción se ha utilizado como mecanismo de busca de legitimación del sistema.

Uno de los ejemplos más claros del poder legitimador de la traducción para el sistema cultural e intelectual gallego (junto al énfasis en su potencial de conscienciación identitaria) se produjo en el primer tercio del siglo XX, con el resurgir de la traducción a gallego en paralelo a la conformación del movimiento político nacionalista alrededor de las Irmandades da Fala y de la Xeración Nós (cf. Millán-Varela 2000). ${ }^{2}$ A manos de los mismos protagonistas, la traducción se concibió como una fuente de recuperación de/para la lengua y cultura gallega, esto es, como una herramienta empleada para intentar solucionar problemas y carencias que se presentan en los ámbitos político, lingüístico y literario. Su valor excedía el proyecto de recuperación de la lengua, para erigirse en fórmula de dignificación y desarrollo de una

2. Las Irmandades da Fala, activas entre 1916 y 1936, fueron una organización nacionalista con programa político con la que el movimiento galleguista asumió por vez primera el monolingüismo en gallego. La Xeración Nós, así llamada por el título homónimo de la revista que publicaban, estaba constituida por un colectivo de intelectuales y escritores que contribuyeron de forma crucial a la revitalización de la literatura gallega y en gallego. 
cultura autóctona que se deseaba conectar directamente con Europa desde un "particular e apaixonado compromiso de descolonizar Galiza política e culturalmente" (Caneda Cabrera 1998: 90). Esta filosofía se refleja en los criterios de selección de las obras, orientados hacia

afianzar os vencellos étnicos, culturais e nacionalistas con outros pobos [...]. A tradución resulta unha actividade empregada principalmente para contribuír á creación dunha identidade galega. Úsase como estratexia modernizadora da lingua e da literatura galega, esforzándose en achegar modelos vangardistas que rompan a inercia da creación galega. (Real Pérez 2000: 27)

El formato de publicación de las traducciones es significativo del papel político que se les otorga. Justamente por esta importancia recibida, entre 1916 y 1936 hubo una profusión de traducciones hacia el gallego que abarcaban no sólo textos literarios, sino también (y sobre todo) textos políticos, de sociología, economía, lengua, arte, crítica literaria o filosofía, como se desprende del exhaustivo estudio sobre este período realizado por Beatriz Real Pérez (2000). Dejando a un lado las (únicamente) seis traducciones literarias publicadas en formato libro, en toda esta época el resto de las más de cien traducciones a gallego vieron la luz las revistas A Nosa Terra y Nós (editando 421 y 144 números, respectivamente), las cuales servían como vehículos políticos de la causa nacionalista gallega.

Tras el parón del tráfico editorial de la traducción que supuso la llegada del régimen dictatorial de Franco, desde finales de los años 50 se inició un activismo político en la resistencia cultural. La mayoría de las (aún minoritarias) traducciones respondían a la iniciativa individual de traductoras/ es, según sus intereses y competencias lingüísticas. Por ejemplo, en 1951 se inició la Biblioteca Antolóxica de Clásicos por iniciativa del Instituto Padre Sarmiento, con la finalidad de demostrar que el gallego era una lengua válida para todos los géneros escritos. En el exilio también se retomó esta actividad, especialmente alrededor de la Editorial Galaxia y su revista Grial, de la editorial Sept y de la colección Bibliófilos Gallegos (cf. Rubio Puga 1999).

Con todo, los cambios políticos posteriores a 1975 permitieron un nuevo giro que, respecto a las políticas editoriales, implicó el inicio de un movimiento de recuperación de la identidad política, lingüística y cultural de Galicia, como muestra el hecho de que

[e]n este último cuarto de siglo se publicaron en gallego más libros que en toda la historia de Galiza, con un incremento casi constante en cifras absolutas que se puede considerar como vertiginoso. Así, de 57 títulos (no todos ellos de carácter literario, claro está, aunque sí mayoritariamente) en 1975 pasamos a 1.145 en 2000, con una subida porcentual de casi un 2000. (Constenla Bergueiro 2004) 
La evolución del tráfico de recepción tuvo una evolución semejante al de la producción editorial global. Se pasó de las 6 traducciones de 1975 a las 129 traducciones del 2000, año en que la traducción constituía el 11,26 por ciento del mercado editorial global. Los datos relativos a 2007 indican que un 18 por ciento de la edición gallega fueron traducciones, incluyendo libro infantil y juvenil, libro de texto, libros de creación literaria, ciencias sociales y humanidades, libros científico-técnicos y libros de ocio (cf. Luna Alonso 2009). Ahora bien, contra esta tendencia en positivo de los últimos años, los estudios más recientes alertan de un futuro definido por una bajada en la producción, en parte debida a las nuevas políticas públicas que desde 2009 contemplan una significativa reducción "das axudas á tradución por parte da Xunta de Galicia, o que tamén provocará que as editoriais, sobre todo as que máis invisten en importación de textos contemporáneos procedentes doutros espazos culturais cara ao noso e viceversa, han reducir as súas apostas nese ámbito" (Luna Alonso 2010: 207).

\section{Las mujeres y/en la traducción a gallego}

A tenor de las particularidades que muestra la traducción en el contexto gallego en este período, el primer tercio y el último cuarto del siglo XX resultan las etapas más productivas en las que examinar las contribuciones de las traductoras.

\subsection{El resurgir del nacionalismo en el primer tercio del siglo $X X$}

El primer tercio del siglo XX, período de conformación del nacionalismo, fue uno de los momentos de mayor publicación de traducciones a gallego (sólo superado por el actual), y también uno de los más estudiados. Someter a una lectura de género el minucioso repaso de Real Pérez (2000: 10-18) por el centenar de traducciones a gallego que en esta etapa vieron la luz en A Nosa Terra y Nós, permite constatar la total inexistencia de traducciones por parte de traductoras en estas revistas, las cuales funcionaban como (prácticamente) único soporte en el que poder publicar traducciones a gallego. Dado que el criterio de la lengua (de la traducción) se considera habitualmente como preferente a la hora de determinar la pertenencia al sistema cultural (lo que, en mi opinión, resulta por lo general acertado), a la vista de estos datos se podría concluir que en esta época no hay traductoras que contribuyan a la conformación del sistema cultural, lingüístico y literario gallego. Efectivamente, esta aseveración resultaría cierta si se interpreta de forma literal. Sin embargo, cabe aclarar que sí existen en este período traductoras gallegas que, pese a 
traducir prácticamente siempre a castellano, muestran una clara conciencia nacional y galleguista. Conviene, pues, analizar las razones que subyacen a esta actitud aparentemente contradictoria para discernir hasta qué punto su labor podría resultar (o no) pertinente y relevante en la caracterización del sistema cultural.

En algunos casos, la elección del castellano podría achacarse a su localización geográfica fuera de Galicia. En otros, a la tendencia bastante habitual durante las fases iniciales de recuperación lingüística de defender una lengua y/o literatura minorizada utilizando como idioma vehicular la lengua normalizada contra la que compite. Pero además, considero que también podría ser resultado de un doble proceso de discriminación de las traductoras que se retroalimenta: por un lado, la opresión que padecen como mujeres y sujetos subalternos de una nación colonizada en la coyuntura sociolingüística de comienzos del siglo XX; y por el otro, la discriminación que sufren como mujeres e intelectuales por parte del nacionalismo gallego de la época.

\subsubsection{La opción lingüística como violencia de género}

Que las traductoras gallegas con una clara conciencia nacional traduzcan a castellano puede interpretarse, en cierta medida, como resultado de la opresión de género que emana de la situación sociolingüística en la que el gallego es la lengua minorizada. Porque, si bien también lo es para los traductores, los estudios sociológicos coinciden con los análisis lingüísticos en interpretar el habla de las mujeres como más próxima a la norma de prestigio, tanto en el interior de una única lengua, como también (y lo que es más relevante para este caso) en la situación de contacto de lenguas. Esto se debería a la necesidad que sienten las mujeres, ante su mayor vulnerabilidad social, de construirse en un modelo no estigmatizado que les permita huir de una posición aún más subordinada de la que ya poseen socialmente. De ahí que, con el fin de evitar el rechazo, las mujeres sean las que antes se asimilan lingüísticamente a la norma prestigiada y a la idea de corrección, adoptando el tipo de lengua (o el idioma, en situación de contacto interlingüístico) que más las legitime (Bourdieu 1991) y rechazando aquello que consideran lingüísticamente marcado como negativo (García Mouton 2003). En la situación diglósica de contacto gallego/castellano, ése era el caso del idioma propio, tradicionalmente vinculado a las clases pobres y campesinas, frente a las clases medias y a la 
burguesía castellana y castellanizante. ${ }^{3}$ De esta presión de los roles de género sobre las mujeres, y en especial sobre las mujeres cultas, deja constancia en 1929 Mari Luz Morales en el escrito "Galega, ipara i-escoita!", en el que reprende a las mujeres cultas por abandonar su lengua propia:

¿Que absurda ideia d'unha 'finura' falsa, dun aristocratismo postizo i-arbitrario te leva a desterrala da intimidade do teu fogar? ¿Acaso non sabes que poser duas lingoas propias, nosas, é o mesmo que ter duas almas? (en Obelleiro 2010, en línea)

El uso preferente del castellano por parte de las mujeres cabría entenderlo así como fórmula que muchas aplicarían para adecuarse al modelo de prestigio, en una estrategia de autodefensa contra su exclusión social. Para Goretti Sanmartín, esta presión social hacia las mujeres para abandonar la lengua menos prestigiada socialmente es resultado de un proceso de violencia de género, aunque

case nunca se acompaña ese discurso [de la violencia de género] coa afirmación de que o feito de erradicaren a lingua a tantas mulleres desta nación tamén responde a unha actuación de violencia sufrida polo colectivo feminino que, como é sabido, soporta unha moito máis forte presión social para se axustar aos modelos sociais da corrección. (Sanmartín 2009: 50)

Dada esta situación, pocas escriben en gallego, y menos aún traducen hacia esta lengua, lo que en parte explicaría su ausencia como traductoras en aquellas publicaciones como A Nosa Terra y Nós. Ahora bien, para lograr una caracterización más íntegra de su ausencia es vital dar un paso más y preguntarse también por la actitud que desde el discurso nacional/ista se mostraba hacia las mujeres y hacia la traducción.

\subsubsection{La exclusión del discurso nacional}

La importancia que la Xeración Nós otorgaba a la traducción como herramienta política la convertía en un asunto político primario (no secundario), y por lo tanto únicamente apto para los patriarcas del nacionalismo (y no para las mujeres). En esta altura, desde la militancia política nacionalista la apreciación que se tenía de las mujeres era la de seres relativos a quienes se instaba a trabajar por los objetivos nacionalistas, por resultarles éstos beneficiosos en tanto que madres-educadoras de generaciones futuras, y en tanto que esposas de emigrantes, campesinos y marineros que verían mejorar su situación con

3. Estudios recientes demuestran como en la actualidad todavía pueden apreciarse, y en muy alto grado, estas actitudes de las mujeres hacia la lengua propia (cf. González Veira y Seoane Bouzas 2010; Moure 2010). 
la llegada al poder del nacionalismo, aunque no por resultarles provechosos a ellas mismas como personas y seres plenos (Rios Bergantinhos 2001: 66).

Las mujeres eran además un objeto clave en el imaginario nacional/ista, en una tendencia que se repite a lo largo del planeta desde tiempos inmemoriales, pues "throughout global history, with few exceptions, women, the feminine, and figures of gender, have traditionally anchored the nationalist imaginary - that undisclosed ideological matrix of nationalist culture" (Heng 1997: 31). Como evidencia Belén Martín Lucas, también en el caso gallego los cuerpos de las mujeres se utilizaron como metáforas de la tierra y de la patria, definiendo en femenino "[a] la tierra en la que el hombre planta su semilla para que dé frutos, y así se concibe también a la tierra, como una madre que cuida, protege y da alimentos a sus hijos" (Martín Lucas 2000: 163). De este modo, se crea una feminización simbólica de la nación como una mujer con la que casarse o a la que amar, junto a una identificación simbólica de la "madre patria" como vínculo maternal (esto último, de una manera especial en aquellas sociedades con un índice alto de emigración, como la gallega). Aunque tanto una como otra pretendan presentarse como un tratamiento honorífico a las mujeres, ambas retóricas funcionan como instrumento de cosificación: en el primer caso, porque "al ser elevada al símbolo de la nación la mujer deja de ser una persona y sujeto autónomo cuyos sentimientos cuentan, para convertirse en un simple instrumento y manera de expresión del deseo masculino" (Nussbaum, en Martín Lucas 2010: 54). Y en el segundo, también la potenciación simbólica de las madres lleva consigo una serie de peligros, pues

lejos de favorecer el progreso feminista lo entorpece, ya que se proyecta la imagen de estar defendiendo a las mujeres al darles un protagonismo simbólico, cuando en realidad se están reafirmando los valores patriarcales que reducen a la mujer al papel de reproductora, de protectora de la comunidad y de transmisora de los valores tradicionales. (Martín Lucas 2010: 55)

En efecto, la no consideración de las mujeres como sujetos políticos plenos se traduce finalmente en el espacio exiguo que les reservaron a las intelectuales en las publicaciones galleguistas A Nosa Terra y Nós, considerados manifiestos de los partidos nacionalistas, en las que tan sólo 8 autoras pudieron publicar en alguna ocasión sus escritos y artículos originales (Rios Bergantinhos 2001: 68) aunque, como argumentaba, ninguna traducción. ${ }^{4} \mathrm{Al}$ ser estas publicaciones prácticamente las únicas que utilizaban el gallego como lengua vehicular,

4. El artículo citado de Mari Luz Morales constituye una excepción, y también un buen ejemplo de cómo el nacionalismo gallego de la época, pese a excluir a las intelectuales de sus publicaciones en gallego, mostraba preocupación por la pérdida de hablantes mujeres (cf. Sanmartín 2002: 100-107). 
con esta actitud los miembros de la Xeración Nós restringían de forma aciaga el acceso de las mujeres a la traducción (y en gran medida, también a la escritura) en la lengua propia.

De este modo, si bien a nivel internacional numerosos estudios demostraron cómo la traducción se consideraba una actividad secundaria frente a la producción autorial que era primaria, en el contexto gallego la importancia dada a la traducción como vehículo político la convertiría más bien en actividad primaria y, por tanto, masculina. Podría así afirmarse que la tradicional consideración de la traducción como actividad femenina por su supuesto carácter secundario respecto al original desaparece a medida que aumenta la importancia que se le otorga. También en relación con esta relevancia, destaca la inexistencia en el sistema cultural gallego de alusiones a la tradicional equiparación metonímica de la doble inferioridad de mujer y traducción que iguala lo femenino con el elemento secundario y derivativo. Con todo, la ausencia de este discurso sexuado y sexista probablemente se deba al hecho de que la personificación en femenino ya estaba "ocupada" con su atribución a la nación, siendo esta retórica un pilar irrenunciable para el propio discurso nacional/ista.

Por extensión, si a nivel internacional la traducción actuó como instrumento liberador al permitir el acceso de estas mujeres al mundo literario como traductoras (a pesar de ser, paradójicamente, mecanismo opresor por condenarlas al margen del discurso), a nivel gallego la lengua castellana serviría como plataforma lingüística en la que las traductoras gallegas pudieron publicar sus traducciones (a pesar de ser el idioma colonizador y al que la presión social y los roles de género empujaban a las mujeres), esto es, como mecanismo para entrar en el mundo literario, incluso si éste era "foráneo".

\subsubsection{Traductoras relevantes de comienzo de siglo}

Ante esta situación, considero vital que en la reconstrucción de la historiografía traductológica gallega no sólo se tenga en cuenta la contribución primordial de estas traductoras de comienzos de siglo que, pese a evidenciar una profunda conciencia nacional/ista, recurrieron al castellano como lengua en la que producir sus traducciones, sino que además se contextualice su elección lingüística en el marco histórico, social y político en el que se localizan, como resultado de un doble proceso de discriminación que se retroalimenta. De no hacerlo, limitándose a afirmar que en este período simplemente no hubo traductoras hacia el gallego, se estará ofreciendo una caracterización parcial y ciertamente inexacta del polisistema cultural. 
Entre los nombres más destacados de autoras cuya faceta traductora todavía no resulta suficientemente reconocida dentro de la historiografía feminista gallega, es de subrayar la labor de Mercedes Vázquez Fernández Pimentel (?1964), quien destacó por sus traducciones publicadas en los años 20 en las revistas Yunque y Estela de poemas del alemán de Albert Sergel, Emma Böhm y Emma Mullenhoff, con unas versiones muy elogiadas por el autor Evaristo Correa en su breve etapa galleguista, afirmando que mostraban una "grande sensibilidade" (Marco 1993: 193).

La vida profesional de la periodista Mari Luz Morales (1889-1980) se desarrolló prácticamente en Cataluña, donde había fijado su residencia y donde se convirtió en la primera mujer en dirigir un periódico estatal, La Vanguardia. Esta localización, sin duda, influiría para que en su caso la lengua de sus traducciones fuese también el castellano. Con todo, Morales estuvo muy vinculada a la vida cultural y política a favor de la cultura gallega (como miembra de la Asociación de Escritores Galegos y del Partido Galeguista, movilizándose a favor del primer Estatuto de Autonomía para Galicia) y también de las mujeres (como secretaria de las Mujeres Galleguistas, durante la guerra). En sus escritos periodísticos en gallego y castellano mostraba esta doble consciencia, escribiendo "textos fervientemente feministas y reivindicativos, también del gallego" (Obelleiro 2010, en línea). Entre las numerosas traducciones que firmó en esta época, conviene subrayar la obra recopilatoria en inglés que adaptó al castellano con el título Obras maestras al alcance de los niños, un volumen de gran trascendencia que fue referente en las escuelas. En los años 20 y 30, tradujo del catalán al castellano relatos de Caterina Albert para el periódico El Sol de Madrid. En esa misma combinación lingüística, vertió al castellano el libro Suburbio de Xavier Benguerel, y algunos poemas del escritor catalán Pere Quart, que entonces era director de las publicaciones de la Generalitat de Catalunya. La calidad de esta traducción le fue agradecida por el mismo escritor, según se recoge en una carta de 1937 conservada en el Ateneu Barcelonès: "La vostra traducció em sembla molt enginyosa i llevat de dues estrofes, altrament intraduïbles (més amb el peu forçat de l'íu) perfecta, fidelísima" (en González Fernández 2009, en línea). Morales fue también pionera en la traducción audiovisual, ejerciendo como traductora y autora de diálogos cinematográficos para Paramount. En medio de una obra prolífica, el perfil periodístico de Morales la llevó a publicar Las románticas, libro en el que traza el perfil biográfico de mujeres excepcionales. Entre ellas figura Madame de Staël, quien en 1816 se había convertido en la primera mujer en publicar un tratado sobre traducción (De l'esprit des traductions), en el que presenta aportaciones realmente innovadoras para la época (cf. Simon 2002; 
Stark 1993). ${ }^{5}$ La obra de Morales constituye, de este modo, el primer (y quizás único) estudio realizado por una gallega sobre la autora del primer tratado sobre traducción escrito por una mujer.

Al lado de estos nombres, hay que mencionar también el de la mugardesa Teruca Bouza Vila (1906-2005), que realizó traducciones muy aclamadas de comedias castellanas a gallego así como traducciones también a gallego de textos teatrales clásicos desde el francés como Le médecin malgré lui ( $O$ médico a paus) de Molière, representada en el teatro Jofre de Ferrol en 1929. Quizás precisamente por su elección lingüística, estas traducciones todavía permanecen inéditas, aunque sin duda su alto valor histórico-documental convertiría su publicación en más que recomendable.

\subsection{De la dictadura a la Ley de Normalización}

La tendencia de que el castellano fuese la lengua de la traducción incluso en el caso de autoras (y autores) con un discurso galleguista se acentuó con la llegada de la dictadura, debido en parte a factores contextuales muy evidentes, como la represión lingüística de los primeros años del régimen o la experiencia del exilio en Hispanoamérica. En este marco se sitúa la labor traductora de la escritora y pedagoga María Barbeito (1880-1970), que tras su expulsión de la docencia con la instauración del régimen franquista en 1936, se dedicó a traducir a autoras y autores franceses a castellano. Entre sus traducciones publicadas se hallan Perros perdidos sin collar de Gilbert Cesbron, El libro de los novios de Marc d'Isolin o La piedra angular de Zoé Oldenbourg. Sin embargo, otras de sus traducciones permanecen todavía inéditas, como la del Livre de ma vie, de Ana de Noailles, de la que hasta el momento tan sólo se ha publicado el prólogo de la traductora, en el que Barbeito analiza la obra, temática y estilo, además de examinar cuestiones traductológicas.

En el exilio desarrolló su labor Amparo Alvajar (1916-1998), que se trasladó a Argentina tras el golpe militar franquista por sus vínculos familiares galleguistas, republicanos y feministas, con un padre político en la II República y con una madre directiva y activista de una asociación republicana de mujeres. Además de su reconocido éxito como autora teatral, recibiendo el Premio Nacional de Literatura en Buenos Aires, Alvajar se dedicó profesionalmente a

5. Contradiciendo la opinión mayoritaria del momento, que ve la traducción como una actividad secundaria y derivativa, de Staël le otorga a la traducción una función revalorizada y positiva, la cual, lejos de empobrecer la literatura extranjera, sirve para destacar su belleza. Con todo, su ensayo apenas aparece mencionado en los estudios panorámicos sobre teoría e historia de la traducción. 
la traducción desde mediados del siglo XX. Su faceta como traductora literaria del francés (Diderot, Flaubert, Bollard), del italiano, del inglés y del portugués le valió la consideración de "mellor tradutora de Hispanoamérica" (Marco 2007: 22). Además, Alvajar fue la primera persona gallega en trabajar como traductora de las Naciones Unidas en Nueva York, de la Unesco en París y de la Organización Mundial del Trabajo en Ginebra. Esta labor la colocó en una posición privilegiada desde la que reflexionó sobre la traducción no literaria, sobre todo en epistolarios como los que mantuvo con Carmen Muñoz, con el marido de ésta Rafael Dieste, así como con su hermana Ana María Alvajar, todos ellos recogidos en diversos trabajos sobre la experiencia vital de la traductora narrados por su propia hermana (cf. Alvajar 2000 y 2002). La contribución traductológica de Alvajar (en castellano) debe enmarcarse en la labor a favor de la cultura gallega realizada desde el exilio, y en la que con mucha frecuencia está presente su conciencia galleguista junto a la nostalgia por una tierra a la que voluntariamente no regresaría jamás: la traductora pasó los últimos anos de su vida en Portugal, "preto da fronteira coa súa amada terra natal, pero sen atreverse a voltar a pisala máis" (Portela Yáñez, en Alvajar 2000: 5). Su labor de conjunto llevó a Luís Seoane a definirla, tal y como se recoge en la contracubierta de Notas sobre Amparo Alvajar, como

unha das mulleres de información intelectual máis traballada da Galicia de hoxe [...] Agora matina traballar novamente para Galicia desde fóra, aínda que non deixou endexamais de traballar para ela, como ocorre con moitos escritores e artistas desa xeración cuxa obra foi feita por circunstancias especiais no estranxeiro e nas peores condicións. (Seoane, en Alvajar 2000)

En definitiva, la debilidad del polisistema provocó que las traducciones a lengua gallega en esta etapa fuesen muy escasas en general, y más todavía en el caso de las traductoras. Esta ausencia resulta especialmente significativa en comparación con las prolíficas aportaciones de las traductoras en otros territorios con lengua propia, como el catalán (cf. Godayol 2006 y 2007).

De hecho, habría que esperar hasta 1967 para que una traductora publicase en formato libro una traducción a gallego. Este papel pionero corresponde a Xohana Torres (1931- ), quien así se convirtió, además, en la primera persona traductora a gallego de textos de literatura juvenil e infantil, fruto de la colaboración entre la editorial catalana La Galera y la gallega Galaxia para traducir literatura infantil entre ambos sistemas (con las colecciones A galea de ouro y Desplegavelas) sin tener que pasar por la literatura central de la comunidad interliteraria (Domínguez 2009: 45). Continuó con su labor pionera hasta convertirse, junto a Carlos Casares y Salvador García Bodaño, en una de las más prolíficas traductoras de literatura infantil y juvenil al gallego en 
la década de los 70 y 80, además de autora en 1968 del primer libro gallego traducido a las cuatro lenguas peninsulares, Polo mar van as sardiñas (Domínguez 2008: 25). Esta constante labor traductora de literatura infantil, acentuada por su labor inaugural en el ámbito gallego, convertiría su nombre en más que apropiado para denominar un posible premio de traducción de literatura infantil y juvenil a lengua gallega. Su creación, por otra parte, resultaría más que deseable, a la vista de la enorme y creciente importancia que posee la traducción de esta literatura en Galicia.

El progresivo (aunque lento y todavía incompleto) avance hacia la normalización del idioma gallego quizás provoque que a partir de los años 70 el idioma constituya de nuevo, con menos fisuras, el criterio principal de pertenencia al sistema cultural. Esto es, aunque en la actual coyuntura pervivan las actitudes de desprecio de las mujeres hacia la lengua propia como resultado de su intento de adecuación a los roles de género, en mi opinión el polisistema cultural está suficientemente evolucionado como para que la elección lingüística constituya el criterio primordial para determinar la pertenencia a dicho polisistema. Lejos de la postura reciente de algunas voces que piden la sustitución del criterio de la lengua por el del lugar de procedencia del autor/a -véase, por ejemplo, el caso de Marta Rivera de la Cruz cuando afirma que "non se debe monopolizar a literatura galega cos autores que escriban nesta lingua" (en Hooper 2003: 111) - a mi juicio el criterio lingüístico resultaría ciertamente idóneo, tanto por basarse en un acto de voluntad (su elección es fruto de una decisión libre) como por su carácter inclusivo (su aprendizaje está abierto a cualquier persona, independientemente de su lugar de nacimiento).

Entre el cada vez mayor número de traductoras que desde la aprobación de la Ley de Normalización Lingüística (1983) traducen a gallego, Teresa Barro (1936- ) merece un mayor reconocimiento. Mostrando un claro compromiso por la dignificación y revitalización del idioma, fue traductora a gallego, junto a Fernando Pérez Barreiro, de Alice in Wonderland, uno de los primeros libros vertidos a la lengua propia tras la mencionada Ley de Normalización. Su versión se convirtió en la primera traducción a gallego que recibía el premio de traducción otorgado por el Ministerio de Cultura (1985), un galardón "highly significant, considering that the Galician translation had to compete with translations into all the languages of the Spanish State" (Millán-Varela 2000: 276). 


\subsection{Algunos apuntes sobre la actualidad: ¿una profesión feminizada?}

En términos generales, los datos avalan hoy la tesis de la feminización de la profesión. No es sólo que la mayoría de las personas matriculadas en los estudios superiores de Traducción en Galicia sean alumnas, sino que esta feminización de la licenciatura también se corresponde con las cifras de las personas miembras de la Asociación Galega de Profesionais da Traducción e Interpretación, compuesta en un 81 por ciento por traductoras. ${ }^{6}$ Sin embargo, como apunta Neal Baxter (2010: 123), en el ámbito de la traducción literaria las cifras de traductoras que publican en las dos editoriales líderes de traducción desciende considerablemente, situándose en un 26,88 por ciento en el caso de la editorial Xerais, y en un 35,29 por ciento en el caso de Rinoceronte Editora. Examinar las relaciones de poder en cuanto al género que se dan dentro del acto de mediación que es toda traducción, permitiría concluir que

as tradutoras si exercen de bonnes à tout faire no ámbito infravalorado da tradución non literaria, mentres que, segundo unha repartición de papeis dos máis habituais, o ámbito prestixiado da tradución literaria fica maioritariamente en mans de tradutores varóns. [...] Mentres hai unha elite de varóns tradutores literarios prestixiados, existe un batallón enorme de mulleres tradutoras anónimas que levan a cabo os labores máis rutineiros e desagradecidos da tradución xeral e técnica. (Baxter 2010: 125)

En esta tendencia parecen desempeñar un papel destacado las políticas editoriales hacia la traducción de las propias industrias literarias gallegas. De acuerdo con las dinámicas que generan, cuando son las editoriales las que toman la iniciativa sobre una traducción, en ocasiones se la confían a escritoras y escritores ya conocidos (dispongan o no de formación y/o experiencia específica en el ámbito de la traducción), tal vez como reclamo para su mejor comercialización. Pero en otras ocasiones, cuando la propuesta surge de la traductor/a, las propias escritoras y escritores son quienes más fortuna tienen a la hora de que las editoriales (sobre todo aquéllas que ya publican sus originales) acepten sus propuestas de verter a gallego obras extranjeras, que posteriormente ellas y ellos mismos se encargan de traducir. ${ }^{7}$ La proporción

6. Cálculo realizado consultando la base de datos incluida en la página web de la AGPTI, disponible en http://agpti.org/galego/asoc_gal.php. Dejando fuera del cómputo a las 6 agencias de traducción, de las 84 personas asociadas, 68 son traductoras y 16 traductores. (Consulta: 02/01/2010).

7. Estas tendencias remiten al viejo debate de si la gente del mundo de las letras está mejor preparada para traducir literatura, o si por el contrario lo más relevante es la experiencia y formación en técnicas de traducción. Aunque no es éste el lugar para abordarlo, resultan muy elocuentes las diferentes posiciones de traductoras y traductores recogidas 
ostensiblemente mayor de escritores que escritoras en el sistema literario gallego (pese a la mayor presencia y reconocimiento que éstas han ido logrando en los últimos años) podría explicar, al menos en parte, esta tendencia de la masculinización de la traducción literaria. Ante esta situación verificable se desencadenan dos comportamientos discriminatorios, pues no sólo quedarían relegadas las traductoras a la traducción profesionalizante menos prestigiosa y (re)conocida, sino que asumir como verdad general la tesis de la feminización de la profesión sin tener en cuenta las relaciones de poder que operan en las dinámicas editoriales, tendría también una funcionalidad análoga al discurso de la "sobrerrepresentación" de lo femenino, dando a entender que ya se ha conquistado la superioridad numérica en la traducción y por tanto actuando como mecanismo despolitizador y de desmovilización social (cf. Freixas 2009 para un examen de las nefastas consecuencias de este discurso en el ámbito literario).

\section{A modo de conclusión}

En el marco del reciente interés por desvelar el papel de la traducción y de las mujeres en la historia de la humanidad, este artículo ha querido contribuir a difundir las aportaciones de las traductoras gallegas del siglo XX, analizando las relaciones de poder presentes en su labor de traducción a la luz del discurso nacional y de género. Teniendo en cuenta las particularidades del caso gallego, considero que es vital reivindicar, en primer lugar, su faceta en tanto que agentes de traducción, la cual hasta la fecha ha pasado sumamente desapercibida en los libros de historiografía feminista gallega. Pero también, en segundo lugar, su aportación en tanto que mujeres, una contribución constantemente obviada en la historiografía gallega de la traducción.

En este sentido, junto a la necesidad de poner en valor la labor de aquellas traductoras que tradujeron a gallego, he querido subrayar la importancia de revisar los criterios que definen la construcción de la historia de la traductología gallega, pues aunque la elección del idioma pueda resultar a priori un criterio válido para establecer la pertenencia al polisistema cultural, en determinadas circunstancias (como las producidas a comienzos del siglo XX, en el momento inicial de configuración del nacionalismo gallego) debería enfocarse de forma más flexible. De esta forma, se podrá ganar una perspectiva más íntegra sobre las dinámicas en marcha en la historia de la traducción y sobre el papel de las traductoras en el sistema cultural gallego. Esta

en un texto periodístico publicado en 2008 en la edición gallega del diario El País (cf. Salgado 2008). 
flexibilidad requeriría que el relato de la historia cultural gallega incluyese y contextualizase el papel desempeñado por estas traductoras que mostraban un claro compromiso nacional/ista, independientemente de que algunas de ellas tradujesen a castellano.

La contextualización implicaría explicar esta dinámica como resultado no sólo de factores como la localización geográfica, el exilio o la situación de extremísima debilidad del sistema literario hasta las últimas décadas del siglo XX, sino también como consecuencia en determinados momentos de un doble proceso de discriminación: la opresión de género en una sociedad diglósica que pone más presión sobre las mujeres para que encajen en los roles sociales y lingüísticos considerados apropiados para ellas (abocándolas a que hagan suya una lengua diferente a la propia de su territorio), y de forma interrelacionada con ello, su expulsión del discurso nacionalista y de las revistas en las que se publicaban prácticamente todas las traducciones a gallego ${ }^{8}$. E implicaría también, respecto a la época actual, desenmascarar cómo el hecho de generalizar el discurso de la feminización de la profesión supone incurrir en una sobrerrepresentación de lo femenino, lo que en última instancia oculta las relaciones de poder que operan en las dinámicas editoriales y que todavía siguen apartando hoy a las traductoras gallegas del ámbito más prestigioso de la traducción literaria.

\section{Bibliografía}

AlvajAR, Ana María. (2000) Notas sobre Amparo Alvajar, exiliada gallega. Sada: Ed. do Castro.

AlvajAR, Ana María. (2002) Solando Lastre. Memorias de Amparo Alvajar. Sada: Ed. do Castro.

BAXTER, Robert Neal. (2010) "Tradución e androcentrismo: quen traduce que(n) e como na Galiza”. En: Mosquera, Xesús (ed.) 2010. Lingua e usos. Lingua e Xénero. A Coruña: Universidade da Coruña. pp. 115-135.

Bourdieu, Pierre. (1991) Language and Symbolic Power. Harvard: Harvard University Press.

CANEDA CABRERA, Teresa. (1998) "Literatura, tradución e reconfiguración da identidade nacional: a 'apropiación' galega do Ulysses". Grial 137. pp. 87-97.

CAstro VÁzQuez, Olga. (2009) "Beauvoir en galego. A tradución d'O segundo sexo". Viceversa 14. pp. 141-147.

8. Esta flexibilidad, sin embargo, no sería pertinente en la actualidad, dada la estructura social de mayor normalización lingüística y cultural. 
Chamberlain, Lori. (1988) "Gender and the Metaphorics of Translation". Signs 13:3. pp. 454-472.

COnStenla Bergueiro, Gonzalo. (2004) "La traducción literaria en gallego: 19752000". En: Camps, Assumpta (ed.) 2004. Ética y política de la traducción en la época contemporánea. Barcelona: Promociones y Publicaciones Universitarias.

Cronin, Michael. (2002) "Jane Wilde, ou l'importance d'être Speranza". En: Delisle, Jean (dir.) 2002. Portraits de traductrices. Ottawa: Les Presses de l'Université d'Ottawa \& Artois Presses Université. pp. 266-290.

Delisle, Jean. (2002a) "Présentation". En: Delisle, Jean (dir.) 2002. Portraits de traductrices. Ottawa: Les Presses de l'Université d'Ottawa \& Artois Presses Université. pp. 1-12.

Delisle, Jean. (dir.) (2002b) Portraits de traductrices. Ottawa: Les Presses de l'Université d'Ottawa \& Artois Presses Université.

DelisLE, Jean. (2002c) "Albertine Necker de Saussure, traductrice de transition, 'sourcière' du romantisme". En: Delisle, Jean (dir.) 2002. Portraits de traductrices. Ottawa: Les Presses de l'Université d'Ottawa \& Artois Presses Université. pp. 117-172.

Domínguez, Mónica. (2008) "Polo mar van as sardiñas y sus traducciones". Madrygal 11. pp. 25-33.

Domínguez, Mónica. (2009) "As primeiras traducións de literatura infantil e xuvenil ao galego: normas de tradución, difusión e recepción". Boletín Galego de Literatura 39-40. pp. 37-60.

FREIXAS, Laura. (2009) La novela femenil y sus lectrices: La desvalorización de las mujeres y lo femenino en la crítica literaria española actual. Córdoba: Universidad y Diputación.

García Mouton, Pilar. (2003) Así hablan las mujeres. Curiosidades y tópicos del uso femenino del lenguaje. Madrid: La esfera de los libros.

Godayol, Pilar. (2006) "Traductores". En: Godayol, Pilar (ed.) 2006. Catalanes del XX. Vic: Eumo. pp. 347-365.

Godayol, Pilar. (2007) "Dona i traducció: del plaer a l'ofici". Quaderns Divulgatius 32. pp. 41-53.

GONZÁlEZ-MiLlán, Xoán. (1995) "Cara a unha teoría da tradución para sistemas literarios 'marxinais'. A situación galega”. [Traducción de Silvia Carril Caldelas y María Sola Bravo]. Viceversa 1. pp. 63-73.

GONZÁlEz FERNÁNDEZ, Helena. (2009) "María Luz Morales naquel país misóxino". Das orixes de marzo (21/07/2009). http://dasorixesdemarzo.wordpress.com/ 2009/07/21/2-maria-luz-morales-correspondencia/ (Consulta: 01/08/2009).

GONZÁlEZ VeIRA, Avia \& Nuria Seoane Bouzas. (2010) "Un achegamento aos preconceptos contra o galego por parte das mulleres a través das súas palabras". En: Mosquera, Xesús (ed.) 2010. Lingua e usos. Lingua e Xénero. A Coruña: Universidade da Coruña. pp. 63-78. 
HENG, Geraldine. (1997) "A great way to fly". En: Alexander, Jacqui \& Chandra Talpade Mohanty (eds.) 1997. Feminist Genealogies, Colonial Legacies, Democratic Futures. London \& New York: Routledge. pp. 30-45.

Hoof, Henri van. (1991) Histoire de la traduction en Occident: France, GrandeBretagne, Allemagne, Russie, Pays-Bas. París: Duculot.

HoOPER, Kirsty. (2003) "Girl, Interrupted: the Distinctive History of Galician Women's Narrative". Romance Studies 21:3. pp. 101-114.

Kelly-Gadol, Joan. (1976) "The Social Relation of the Sexes: Methodological Implications of Women's History". Signs 4:1. pp. 809-823.

KORD, Susanne. (1994) "The innocent translator: Translation as pseudonymous behaviour in eighteenth-century German women's writing". The Jerome Quarterly 9. pp. 11-13.

LAFARGA, Francisco. (2005) "Sobre la historia de la traducción en España: contextos, métodos, realizaciones”. Meta 50:4. pp. 1133-1147.

LAMBERT, José. (1993) "History, Historiography and the Discipline. A Programme". En: Gambier, Yves \& Jorma Tommola (eds.) 1993. Translation and Knowledge. Actes du 4e Symposium Scandinave sur la théorie de la traductionTurku 1992. Turku: Grafia Oy \& University of Turku. pp. 3-26.

LEFEVERE, André. (1992) Translation/History/Culture: A Sourcebook. London \& New York: Routledge.

Lerner, Gerda. (1979) The Majority Finds its Past: Placing Women in History. Oxford \& New York: Oxford University Press.

Luna Alonso, Ana. (2009) "Traducións ao galego no 2007". Viceversa 14. pp. 193-206.

Luna Alonso, Ana. (2010) "Traducións ao galego no ano 2008". Viceversa 15. pp. 205-223

MARCO, Aurora. (1993) As precursoras. A Coruña: Biblioteca Gallega y La Voz de Galicia.

Marco, Aurora. (2007) Dicionario de Mulleres Galegas. Vigo: A Nosa Terra.

MARTín LuCAS, Belén. (2000) "Mujer y nación: construcción de las identidades". En: Suárez Briones, Beatriz; Belén Martín Lucas \& María Jesús Fariña Busto (eds.) 2000. Escribir en femenino. Poéticas y políticas. Barcelona: Icaria. pp. 163-178.

MARTín LUCAS, Belén. (2010) "La madre patria: de las metáforas nacionalistas a la violación como crimen de guerra". En: Martín Lucas, Belén (comp.) Violencias (in)visibles: intervenciones feministas frente a la violencia patriarcal. Barcelona: Icaria. pp. 47-68.

Millán-VArela, Carme. (2000) "Translation, Normalisation and Identity in Galicia(n)". Target 12:2. pp. 267-282. 
Moure, Teresa. (2010) "Falarmos galego as mulleres, por que non?". En: Mosquera, Xesús (ed.) 2010. Lingua e usos. Lingua e Xénero. A Coruña: Universidade da Coruña. pp. 147-169.

Noia CAmpos, Camiño. (1995) "Historia da traducción en Galicia no marco da cultura europea”. Viceversa 1. pp. 13-62.

Obelleiro, Paola. (2010) "En memoria de la gran señora de la prensa y escritura”. El País, 21/03/2010. http://www.elpais.com/articulo/Galicia/memoria/ gran/senora/prensa/escritura/elpepiautgal/2010032lelpgal_10/Tes (Consulta: 22/03/2010).

REAl PÉREZ, Beatriz. (2000) "A tradución e os textos traducidos ó galego no período 1907-1936". Viceversa 6. pp. 9-36.

Rios Bergantinhos, Noa. (2001) A Mulher no nacionalismo galego (1900-1936). Santiago de Compostela: Laiovento.

RoBinsOn, Douglas. (1995) “Theorizing Translation in a Woman's Voice: Subverting the Rethoric of Patronage, Courtly Love and Morality". The Translator 1:2. pp. 153-175.

Rubio PugA, Mª Luz. (1999) As traducións publicadas en lingua galega desde 1953 a 1997. Trabajo de fin de carrera inédito. Facultad de Traducción. Universidade de Vigo.

SALGADO, Daniel. (2008) "A tradución perde a inocencia. As tradutoras aseguran que cambiar libros de idioma implica a ideoloxía”. El País, 11/04/2008. http:// www.elpais.com/articulo/Galicia/traducion/perde/inocencia/elpepiautgal/ 20080411elpgal_20/Tes (Consulta: 30/06/2008).

SÁNCHEZ, Dolores. (2010) "Traducción y contexto: el paratexto de la traducción de una obra científica polémica (España, principios del siglo XX)". Ponencia pronunciada en el VII International Conference on Translation: The Paratextual Elements in Translation, Universitat Autònoma de Barcelona, 21-22 junio 2010.

SANMARTín REI, Goretti. (2002) Lendo nas marxes. Lingua e compromiso nos paratextos (1863-1936). A Coruña: Espiral Maior.

Sanmartín ReI, Goretti. (2009) Nos camiños do entusiasmo. Calidade da lingua e planificación. Vigo: Xerais.

SimON, Sherry. (2002) "Germaine de Staël and Gayatri Spivak: Culture Brokers". En: Tymoczko, Maria \& Edwin Gentzler (eds.) 2002. Translation and power. Amherst \& Boston: University of Massachusetts. pp. 122-140.

STARK, Suzanne. (1993) "Women and Translation in the Nineteenth Century". New Comparison 15. pp. 33-44.

Wolf, Michaela. (2005) “The Creation of A Room of One's Own. Feminist Translators as Mediators Between Cultures and Genders”. En: Santaemilia, José (ed.) 2005. Gender, Sex and Translation. The Manipulation of Identities. Manchester: St. Jerome. pp. 15-25. 